\title{
Nociones de género y empoderamiento de las niñas y las mujeres en la escuela. Conceptos y reflexiones"
}

\author{
Notions of Gender and Empowerment of Girls and Women in School. \\ Concepts and Reflections
}

\section{Noções de gênero e empoderamento de meninas e mulheres na escola. Conceitos e reflexões}

\author{
Sorangela Miranda Beltrán** \\ Universidad Externado de Colombia, Facultad de Ciencias de la Educación \\ Colombia \\ sorangela.miranda@uexternado.edu.co \\ https://orcid.org/0000-0002-0886-2647
}

\begin{abstract}
Resumen
El presente artículo enfatiza sobre la relevancia de potencializar el protagonismo social de las niñas y las mujeres, así como la superación de las barreras, los estereotipos y las diferentes formas de discriminación asociadas a la condición de género, que dificultan su plena participación en condiciones de equidad e igualdad de oportunidades, categoría que al ser retomada como una construcción social, posiciona a la escuela como un escenario propicio para el empoderamiento, la participación y el fortalecimiento de la cultura democrática.
\end{abstract}

Palabras clave: cultura democrática, empoderamiento, escuela, igualdad de género.

* El presente artículo se deriva del trabajo de elaboración del marco teórico en el proyecto de tesis doctoral que adelanta la autora, el cual se desarrolla el marco de la línea de investigación en Gestión Directiva y Pedagógica de las Instituciones Educativas de la Facultad de Ciencias de la Educación de la Universidad Externado de Colombia. 


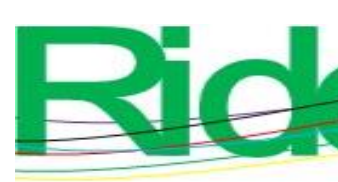
Revista Iberoamericana para la
Investigación y el Desarrollo Educativo
ISSN $2007-7467$

\section{Abstract}

This article emphasizes the importance of girls and women enhancing their social role and overcoming the barriers of stereotypes and the different forms of discrimination associated with gender that hinder their full participation in conditions of equity and equal opportunities. By resuming the category of gender as a social construction, it positions the school as an auspicious setting for empowerment, participation and the strengthening of democratic culture.

Keywords: democratic culture, empowerment, school governance, gender equity.

\section{Resumo}

Este artigo enfatiza a importância de meninas e mulheres valorizarem seu papel social e superar as barreiras dos estereótipos e das diferentes formas de discriminação associadas ao gênero que impedem sua plena participação em condições de eqüidade e igualdade de oportunidades. Ao retomar a categoria de gênero como construção social, posiciona a escola como espaço auspicioso para o empoderamento, a participação e o fortalecimento da cultura democrática.

Palavras-chave: cultura democrática, empoderamento, escola, igualdade de gênero.

Fecha Recepción: abril 2020

Fecha Aceptación: septiembre 2020

\section{Introducción}

El objetivo cinco de La Agenda 2030 y los Objetivos de Desarrollo Sostenible (Organización de las Naciones Unidas [ONU], 2018), delinea un conjunto de acciones orientadas a la igualdad entre los seres humanos, con un firme compromiso de empoderar a las niñas y a las mujeres para potenciar sus capacidades de protagonismo y liderazgo individual y colectivo, en aras de la superación de todas las desventajas asociadas al género, mediante la promoción de procesos participativos, teniendo como referentes la autonomía, la toma de decisiones y el reconocimiento de su injerencia en las diferentes esferas sociales.

La eliminación de las barreras que le impiden a las niñas y a las mujeres la vivencia de los derechos humanos como base fundamental para la vida democrática ha adquirido un lugar importante en el ámbito mundial. El empoderamiento se ha posicionado como la ruta para la ruptura de los estereotipos y ha dado pie a una visión reflexiva, crítica y 


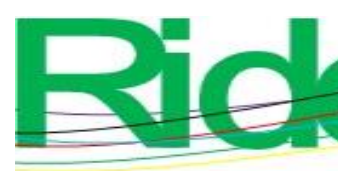

Revista Iberoamericana para la Investigación y el Desarrollo Educativo ISSN $2007-7467$

transformadora que debe ser la constante para revalorar el sentido de vida, el aporte y la lucha de las mujeres por su emancipación (ONU, 2018).

Hacer que las niñas y las mujeres fortalezcan sus posibilidades de poder e independencia en todas las dimensiones del desarrollo social y humano es una tarea que implica, entre otros aspectos, erradicar las formas de violencia que existen contra ellas, las prácticas nocivas que atentan contra su bienestar e integridad y la eliminación de la discriminación que las pone en desventaja en términos económicos, culturales, políticos y públicos (ONU, 2018). Para ello, se hace necesaria la implementación de acciones que contribuyan en garantizar una participación plena en igualdad de oportunidades, el acceso a la educación con calidad y una mayor visibilización en el ámbito económico, participativo (Organización de las Naciones Unidas para la Educación, la Ciencia y la Cultura [Unesco], s. f.).

En este contexto y frente a dicho reto, que involucra la coordinación de dichas acciones, voluntades y esfuerzos de los diferentes actores sociales, el presente artículo se propone reflexionar sobre dos aspectos puntuales: las nociones del concepto de género prevalecientes en la sociedad, que continúan siendo determinantes en las actitudes, ideologías y pensamientos asociados a la feminidad y masculinidad, así como la concepción de la escuela como un territorio donde se legitiman estereotipos que condicionan las posibilidades de participación para las niñas y las mujeres, pero también, donde se pueden suscitar vivencias que potencien la ciudadanía, la democracia, la diversidad y la inclusión que aporten al empoderamiento y reafirmen el papel protagónico de las mujeres en las sociedades.

\section{El concepto de género como constructo social}

La categoría de género es amplia, compleja y de múltiples visiones. Normalmente, es asumida como una construcción social y cultural respecto a las diferencias sexuales (Montecino, 2007 citado en Moreno, Soto, González y Valenzuela, 2017). Así, configura distintas ideas, prácticas y representaciones y da cuenta de aspectos diferenciales entre los hombres y las mujeres, lo que, en palabras de Moreno et al. (2017), conduce a la simbolización de lo que es inherente a los hombres (masculino) y a las mujeres (femenino). En este sentido, reconocer el carácter simbólico del concepto de género implica entender cómo este hace parte de la cultura y la trascendencia que adquiere en las interacciones y en los diversos campos del desarrollo social y humano. 


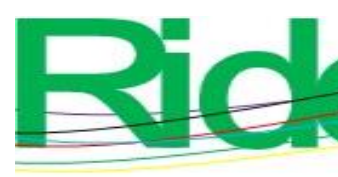

Revista Iberoamericana para la
Investigación y el Desarrollo Educativo
ISSN $2007-7467$

De este modo, se infiere que la categoría de género contiene una amplia carga social y una injerencia significativa en las maneras de proceder y actuar de los individuos, configurando las formas mediante las cuales, tanto los hombres como las mujeres se relacionan y proceden, a partir de patrones sociales que se adhieren a las creencias, los lenguajes y los prejuicios existentes frente a los géneros; en síntesis, generalizan comportamientos y maneras de proceder y relacionarse (Flores, Espejel y Martel, 2016).

Las conductas y las formas de relación materializan los estereotipos que se tienen sobre el género, reflejándose en tratos desiguales a partir del sexo de las personas, desde razonamientos que asocian elementos del ámbito público y privado a los roles masculino y femenino, respectivamente. Las niñas y las mujeres parten socialmente de una posición de desventaja con respecto a su contraparte, conceptualizaciones que se han ido entretejiendo en el devenir histórico-social (Hernández y Treviño, 2016) para demostrar cómo las perspectivas de género o los elementos de religión, ideología, condiciones económicas o características raciales, transversalizan las comprensiones que se tienen sobre este constructo social (Moreno, Sepúlveda y Restrepo, 2012 citados en Flores et al., 2016).

Aunado a lo anteriormente expuesto, las problemáticas de carácter social, económico, cultural, educativo, entre otras, evidenciadas en las condiciones de pobreza, falta de reconocimiento político y vulnerabilidad de las niñas y las mujeres, perpetúan los imaginarios que restringen las posibilidades de desarrollo y pleno goce de los derechos, lo que reafirma los supuestos que configuran las diversas relaciones que se establecen en los espacios y los entornos, que colocan a las niñas y a las mujeres en subordinación, lo que imposibilita la superación de las brechas que durante siglos han caracterizado los aspectos relacionados con el género, la violencia política y otras dimensiones sociales (Lena y Restrepo, 2016).

Bajo esta óptica, la desigualdad se ve ampliamente reflejada en los prejuicios existentes hacia las niñas y las mujeres, donde los estereotipos y los elementos discriminatorios prevalecen (Guzmán, 2017), lo que reafirma las visiones que históricamente se han construido alrededor del género y que dan cuenta de la forma disímil sobre cómo se brindan las oportunidades, sin reconocer el carácter equitativo inexistente en los escenarios políticos, culturales y sociales, lo que se traduce en la subordinación a la que las niñas y las mujeres son sometidas desde una perspectiva que desnaturaliza e invisibiliza sus posibilidades como sujetos políticos. 


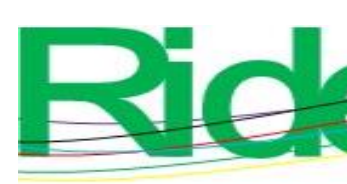

Revista Iberoamericana para la Investigación y el Desarrollo Educativo ISSN $2007-7467$

Lo anterior, como lo expresa Flores et al. (2016), permea los valores culturales, las conductas y las formas de relacionarse reproduciendo actuaciones enmarcadas en el sexismo, la violencia y los variados aspectos que inciden directamente en la creencia del rol de género asignado al sexo o a las características biológicas que conducen a perpetuar los estereotipos a tal grado que toda posibilidad de cuestionar la manera como, desde el ámbito social, se invalida a la niña y a la mujer, reduciéndolas en forma considerable, lo que refuerza la existencia de barreras para que éstas desempeñen un rol activo y de empoderamiento social.

Estos aspectos referidos a las mujeres en el marco de la perspectiva de género se han ido incorporando a las agendas políticas del mundo en las últimas décadas. Las reflexiones sobre esta temática han adquirido la relevancia que se merecen, posicionándose como tema central en los debates políticos y educativos mundiales. Desde 1975, cuando se realizó la Conferencia Mundial del Año Internacional de la Mujer, a la fecha, se han llevado a cabo, por parte de los gobiernos y de los diversos actores sociales, importantes esfuerzos para eliminar toda forma de discriminación contra las niñas y las mujeres; desde entonces, se ha ido avanzando en tópicos relevantes respecto a la igualdad de género, procurando finalizar con todas aquellas prácticas de discriminación, a partir de un escenario político orientado a proveer las herramientas legales y fortalecer los procesos educativos para erradicar todas las formas de violencia que han impedido a las niñas y a las mujeres un desarrollo pleno (Guzmán, 2017).

Este último aspecto, el referido a los procesos educativos, identifica que la familia y la escuela son agentes relevantes de socialización que contribuyen a forjar los valores, los códigos y las diferentes construcciones socioculturales desde los que se asimilan y transmiten los roles y estereotipos a partir de los cuales se conciben y se configuran los conceptos de hombre y de mujer (Santos, 1997; Flores, 2005, ambos citados en Flores et al., 2016). Se trata de un proceso de legitimación sobre las nociones de género a nivel simbólico, de valores y de creencias.

En lo que concierne al papel de la escuela como agente de legitimación y escenario pleno de posibilidades de empoderamiento y emancipación para las niñas y las mujeres, se presentará a continuación la siguiente discusión. 


\section{La escuela: formas de legitimación y posibilidades de empoderamiento para las niñas y las mujeres}

De acuerdo con Flores et al. (2016), la escuela es uno de los dispositivos de reproducción de formas que evidencian las desigualdades, las violencias y la invisibilización de las niñas y las mujeres, realidad que se pone de manifiesto en la cotidianidad del ámbito escolar, en las relaciones que se establecen en el aula, los ritos y las creencias que transversalizan la cultura organizacional y la manera en que se enseña y se aprende en los momentos formales e informales.

Las perspectivas asociadas a estereotipos permean las relaciones que se configuran en la escuela y que, desde este escenario, pueden llegar a adquirir un tamiz natural. Desde lo simbólico, la educación como institución legitima, a través de la estructura curricular, los procesos de formación, los ambientes de aula, las formas de interacción y los sentidos que se le dan al género: existe una reafirmación de prejuicios, modelos mentales e ideas frente a los roles femenino y masculino, sí como de las atribuciones que a cada uno de ellos se les confiere (Moreno et al., 2017).

En esta complejidad, se desarrolla un clima escolar en que los distintos agentes de la comunidad educativa promueven relaciones que estructuran sus nociones sobre género, enmarcadas en ambientes físicos, sociales, afectivos y académicos (González, 2013 citado en Flores et al., 2016). Esta situación demanda el desarrollo de acciones pedagógicas para descentrar las concepciones operativas de la escuela como reproductora de discursos sociales arraigados en supuestos que transitan en la anulación de los principios de igualdad y equidad.

No obstante, al constituir la escuela un foco de legitimación y afianzamiento de estereotipos, también, por su propia naturaleza y fines de formar seres humanos integrales, puede ser un agente transformador a partir de acciones tendientes a romper las conductas sexistas y propiciar de esta manera espacios participativos donde la reflexión, la conciencia crítica y el reconocimiento del otro, se establezcan como la base fundamental para la vivencia de la democracia y los derechos humanos.

En este sentido, se extiende una invitación para la escuela y todos los componentes que integran el sistema educativo para aunar esfuerzos y coordinar estrategias fundamentadas en maneras alternativas de entender el mundo y que ayuden a comprender los asuntos de género más allá de los estereotipos y comportamientos que han privilegiado la jerarquización, la inhibición y la perpetuación del carácter subordinado de lo femenino. Para construir 


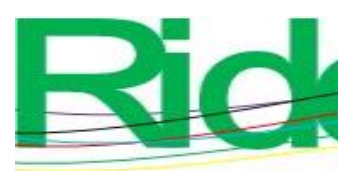

Revista Iberoamericana para la Investigación y el Desarrollo Educativo ISSN $2007-7467$

experiencias conjuntas (entre los niños, los hombres, las niñas y las mujeres) que consoliden ideas y comportamientos basados en la dignidad, el reconocimiento del otro, los derechos humanos, la equidad, la igualdad de oportunidades y la visibilización de los liderazgos, es necesario empezar a promover formas de pensar y entender la cultura democrática, basadas en la posibilidad de participar y ejercer la ciudadanía, alentando el protagonismo, la emancipación y el empoderamiento de las niñas y las mujeres dentro de la comunidad educativa.

Estas consideraciones llevan a pensar que el rompimiento de los estereotipos y todas las formas de discriminación relacionadas con el género, pueden ser posibles cuando se fortalece la formación en ciudadanía y cultura democrática por medio de acciones emancipadoras que orienten a las niñas y a las mujeres hacia el reconocimiento de la diversidad, la práctica de los derechos humanos y los espacios participativos en los que todas y todos puedan ser parte de las decisiones que convocan a las comunidades, tales como el gobierno escolar y sus instancias representativas.

La base democrática debe ser entonces, uno de los escenarios para fortalecer los Proyectos Educativos Institucionales de las escuelas, direccionados hacia un clima organizacional donde prevalezca la sana convivencia mediante la promoción de modos de coexistir que reflejen comportamientos basados en el respeto, el empoderamiento, la eliminación de las prácticas sexistas, la apertura al pensamiento crítico y la gestión del liderazgo escolar. De esta manera, se podrán favorecer las miradas reflexivas que generen y sumen voces para superar, de manera contundente, la estigmatización, las creencias y los patrones comportamentales asociados a la desigualdad de género.

Específicamente, en lo que respecta a las niñas y a las mujeres, se propone enfatizar en procesos participativos que, desde el ámbito escolar, conciban e implementen maneras de intervenir y consolidar el concepto de empoderamiento, construido en la idea del interés común y en la toma de decisiones apoyadas en el bienestar de todos como fuente de la formación política, consolidando un proyecto de estructuración que, desde la perspectiva de género, promueva aprendizajes donde se valore y afiance la trascendencia emancipadora de las estudiantes, las maestras, directivas, madres de familia y demás niñas y mujeres que integran la comunidad educativa. 


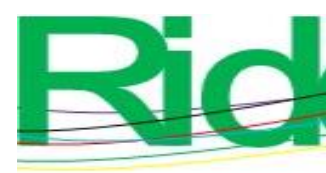

Revista Iberoamericana para la Investigación y el Desarrollo Educativo ISSN 2007-7467

- Situar la participación de los hombres y las mujeres en una cultura y un clima escolar basado en el principio de igualdad de oportunidades y el reconocimiento del otro, desde la perspectiva de la individualidad y su trascendencia en lo colectivo.

- $\quad$ Asumir los retos éticos que Náñez y Capera (2017) describen como parte fundamental de los procesos de formación ciudadana presentes en la escuela. En temas de género, la lógica del discurso debe ser coherente con las prácticas y las acciones concretas que permitan materializar en el sentir, en el ser y en el actuar las construcciones y representaciones mentales frente a la igualdad de oportunidades y equidad de las niñas y la mujeres, permeando las concepciones de los diferentes actores sociales respecto a las formas participativas, exigiendo de ellos transformaciones profundas y radicales en las maneras de significar la construcción social sobre los géneros, reflejándose en la esfera colectiva y en el ejercicio democrático.

- Formar competencias, entendidas como la combinación de los conocimientos y los atributos personales que integrados promuevan características, habilidades y saberes que facilitan el cambio, generando condiciones que conducen a la estabilidad y flexibilidad en los diferentes contextos (Mimbrero, Pallares y Cantera, 2017), cuya aplicación en la perspectiva de género, supone para este caso, la apuesta por la reivindicación de las niñas y las mujeres, promoviendo prácticas sociales que incidan en las realidades para construir visiones completas y transformadoras que superen las barreras de la desigualdad y la discriminación.

\section{Reflexiones finales}

En el escenario educativo actual, la escuela tiene importantes retos para potenciar los ejercicios democráticos y participativos, gracias a los cuales, las niñas y las mujeres puedan romper los estereotipos, empoderarse y ejecutar un rol activo dentro de las comunidades educativas, mediante la apropiación de múltiples aprendizajes que les permitan reivindicar su voz en la toma de decisiones para superar las desigualdades y propiciar una cultura de reconocimiento de la diversidad y transformación social, basada en el respeto por la diferencia, la equidad y la inclusión. 


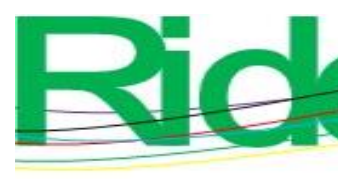

Revista Iberoamericana para la
Investigación y el Desarrollo Educativo
ISSN $2007-7467$

En este sentido, empoderar a las niñas y a las mujeres en la escuela se trata no sólo de garantizar su participación en la toma de decisiones en la institución, sino también de identificar y analizar críticamente aquellos factores que puedan contribuir en el fortalecimiento de su conciencia crítica y emancipación de su pensamiento a través de currículos y procesos educativos que promuevan prácticas sociales generadoras de las transformaciones necesarias en cuestiones de género.

Las acciones para concretar una cultura democrática, de empoderamiento y emancipación de la niña y la mujer en la escuela, deben partir de la concepción de los Proyectos Educativos Institucionales como orientadores de vivencias para consolidar las perspectivas sobre la inclusión, la práctica de derechos y los deberes, así como de la interacción para el crecimiento personal y colectivo que, parafraseando a Osoro y Castro (2017), lleven a asumir a la escuela como un espacio de vida, en el cual se afiance la identidad, el rol protagónico de cada sujeto, las posibilidades de la comunicación y el pensamiento colectivo en el marco de la pedagogía de la participación, la cultura para la paz y la diversidad.

En esta tarea existe un llamado fundamental para que la escuela continúe resignificándose alrededor de la construcción de ciudadanía, la participación y los derechos humanos y avance en la superación de aquellos obstáculos que dificultan la fluidez de una cultura democrática para continuar forjando caminos en los cuales el empoderamiento, el reconocimiento de las niñas y las mujeres, así como la vivencia participativa, contribuyan a canalizar la formación integral y abonen a la discusión productiva, con el fin de que las perspectivas inclusivas sigan adquiriendo valor y se consoliden como la base de la democracia escolar. 


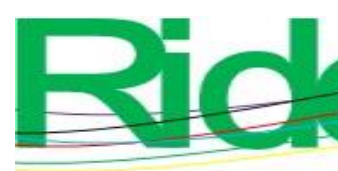

Revista Iberoamericana para la Investigación y el Desarrollo Educativo ISSN 2007 - 7467

\section{Referencias}

Bard, G. (2016). Culturas políticas. (Re)significando la categoría desde una perspectiva de género. Revista Mexicana de Ciencias Políticas y Sociales, 61(227), 137-166. Recuperado de http://www.scielo.org.mx/scielo.php?script=sci_abstract\&pid=S0185$19182016000200137 \& \operatorname{lng}=\mathrm{es} \& n r m=$ iso.

Cubides, H. (2001). Gobierno escolar: cultura y conflicto político en la escuela. Nómadas, (15), 10-23. Recuperado de http://nomadas.ucentral.edu.co/index.php/inicio/34conflicto-educacion-y- Revisiones teóricas.paradi participacióArtículosmamjjjjencia-cultural-nomadas-15/500-gobierno-escolarcultura-y-conflicto-politico-en-la-escuela.

Flores, A., Espejel, A. \& Martell, L. (2016). Discriminación de género en el aula universitaria $\mathrm{y}$ en sus contornos. Ra Ximhai, 12(1), 49-67. Recuperado de https://dialnet.unirioja.es/servlet/articulo?codigo=6463995.

Guzmán, J. (2017): Los Indicadores de Género. La Ruta Hacia la Igualdad. En: Revista Internacional de Ciencias Sociales y Humanidades, SOCIOTAM, XXVII(2), 133147. Universidad Autónoma de Tamaulipas Ciudad Victoria, México. Recuperado de: https://www.redalyc.org/jatsRepo/654/65456039007/html/index.html

Hernández, E., \& Treviño, R. (2016). Perspectiva de Género en la Investigación Urbana. Vivat Academia, (134), 47-56.

Lena, M., \& Restrepo, J. (2016): Género y violencia política en América latina. Conceptos, debates y soluciones Política y Gobierno, XXIII(1), 127-162. Centro de Investigación y Docencia Económicas, A.C. Distrito Federal, México. Recuperado de: https://www.redalyc.org/pdf/603/60343614006.pdf

Marín, F., Riquett, M., Pinto, M., Romero, S. \& Paredes, A. (2017): Gestión participativa y calidad educativa en el contexto del Plan de Mejoramiento Institucional en Escuelas Colombianas Opción, 33(82), 344-365. Universidad del Zulia Maracaibo, Venezuela. Recuperado de: https://dialnet.unirioja.es/servlet/articulo?codigo=6233631

Mimbrero, C., Pallarés, S. \& Cantera, L. (2017). Competencias de Igualdad de Género: Capacitación para la Equidad Entre Mujeres y Hombres en las Organizaciones. Athenea Digital. Revista de Pensamiento e Investigación Social, 17(2), 265-286. 


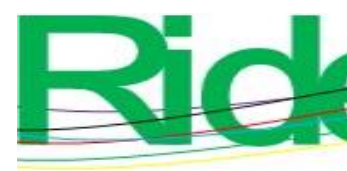

Revista Iberoamericana para la
Investigación y el Desarrollo Educativo
ISSN $2007-7467$

Recuperado de https://atheneadigital.net/article/view/v17-n2-mimbrero-pallarescantera.

Moreno, K., Soto, R., González, M. \& Valenzuela, E. (2017). Rompiendo con los estereotipos: Una experiencia educativa con enfoque de género en una escuela básica. REXE. Revista de Estudios y Experiencias en Educación, 16(32), 165-174. Recuperado de http://www.rexe.cl/ojournal/index.php/rexe/article/view/462.

Ñáñez, J. \& Capera, J. (2017). Participación política y gobierno escolar en las instituciones educativas de Ibagué. Espacios Públicos, (48), 151-171. Recuperado de https://www.researchgate.net/publication/321480148_Participacion_politica_y_gobi erno_escolar_en_las_instituciones_educativas_de_Ibague.

Organización de las Naciones Unidas [ONU]. (2018). La Agenda 2030 y los Objetivos de Desarrollo Sostenible. Una oportunidad para América Latina y el Caribe. Santiago, Chile: Organización de las Naciones Unidas. Recuperado de https://repositorio.cepal.org/bitstream/handle/11362/40155/24/S1801141_es.pdf.

Organización de las Naciones Unidas para la Educación, la Ciencia y la Cultura [Unesco] (s. f.). Educación e igualdad de género. Recuperado de https://es.unesco.org/themes/educacion-igualdad-genero.

Osoro, J. \& Castro, A. (2017). Educación y Democracia: La Escuela Como Espacio de Participación. Revista Iberoamericana de Educación. 75(2). Recuperado de https://rieoei.org/RIE/article/view/2635.

Pérez, L. \& Ochoa, A., (2017): La Participación de los Estudiantes en una Escuela Secundaria. Retos y posibilidades para la formación ciudadana. Revista Mexicana de Investigación Educativa, 22(72), 179-207. Recuperado de http://www.scielo.org.mx/scielo.php?script=sci_abstract\&pid=S140566662017000100179\&lng=es\&nrm=iso.

Vivas, A. (coord. ${ }^{a}$ ) ( 2017). Plan Nacional Decenal de Educación 2016-2026. El camino hacia la calidad y la equidad. Colombia: Gobierno de Colombia. Recuperado de http://www.plandecenal.edu.co/cms/media/herramientas/PNDE\%20FINAL_ISBN\% 20web.pdf. 


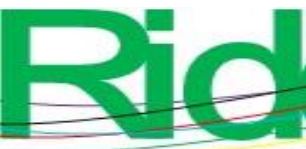

** Sorangela Miranda Beltrán

Postdoctora en Gestión y Administración de Planteles Educativos por el Centro de Estudios e Investigaciones para el Desarrollo Docente Cenid (México), Doctora en Ciencias Sociales y Humanas por la Pontificia Universidad Javeriana (Colombia), Magíster en Administración y Supervisión Educativa por la Universidad Externado de Colombia y Licenciada en Educación Especial por la Universidad Pedagógica Nacional (Colombia). Actualmente, además de desempeñarse como docente en la Maestría en Educación y en la Maestría en Evaluación y Aseguramiento de la Calidad de la Educación, ambas de la Universidad Externado de Colombia, cursa el doctorado en Ciencias de Educación en la Universidad Cuauhtémoc (México). 